\title{
Assessment of mitral valve regurgitation by cardiovascular magnetic resonance imaging
}

\author{
Pankaj Garg ${ }^{1 *}$, Andrew J. Swift ${ }^{1}$, Liang Zhong ${ }^{2}$, Carl-Johan Carlhäll ${ }^{3}$, Tino Ebbers ${ }^{3}$, \\ Jos Westenberg ${ }^{4}$, Michael D. Hope ${ }^{5}$, Chiara Bucciarelli-Ducci', Jeroen J. Bax ${ }^{7}$ and \\ Saul G. Myerson ${ }^{8}$
}

Abstract | Mitral regurgitation (MR) is a common valvular heart disease and is the second most frequent indication for heart valve surgery in Western countries. Echocardiography is the recommended first-line test for the assessment of valvular heart disease, but cardiovascular magnetic resonance imaging (CMR) provides complementary information, especially for assessing MR severity and to plan the timing of intervention. As new CMR techniques for the assessment of MR have arisen, standardizing CMR protocols for research and clinical studies has become important in order to optimize diagnostic utility and support the wider use of CMR for the clinical assessment of MR. In this Consensus Statement, we provide a detailed description of the current evidence on the use of CMR for MR assessment, highlight its current clinical utility, and recommend a standardized CMR protocol and report for MR assessment.

Mitral regurgitation (MR) is a common valvular heart disease and is the second most frequent indication for heart valve surgery in Western countries ${ }^{1}$. Almost 9\% of the general population aged $>75$ years in the USA have $\mathrm{MR}^{2}$. Cardiac imaging is crucial for diagnosis, identifying the cause of the disease, monitoring disease progression and planning definitive treatment for $\mathrm{MR}^{3}$. Echocardiography remains the first-line and most widely available imaging test for the assessment of MR. Cardiovascular magnetic resonance imaging (CMR) has also emerged in the past 20 years as a robust, noninvasive imaging modality for the assessment of patients with $\mathrm{MR}^{4}$. CMR offers a comprehensive evaluation of $\mathrm{MR}$ and its effects on the heart by providing precise volumetric assessment (using cine images) and myocardial scar or fibrosis assessment (using the late gadolinium enhancement (LGE) technique). Therefore, CMR is often complementary to echocardiography in informing the clinical management of $\mathrm{MR}^{4}$.

As more evidence is gathered for the use of CMR in the assessment of $\mathrm{MR}$, clinicians and researchers need a standard CMR protocol to follow in order to improve the consistency of mitral valve assessment. A consistent approach will further improve the clinical translation and adoption of CMR for the assessment of the mitral valve and MR. Although evidence-based data from randomized clinical trials are limited for MR assessment, in this
Consensus Statement we highlight the role of CMR in the assessment of MR and provide recommendations for a standardized protocol and reporting method.

\section{Methods}

A panel of individuals with vast expertise in $M R$ assessment by standard and emerging methods of CMR gathered in a closed group meeting titled 'Mitral Valve Regurgitation Assessment by Cardiac Magnetic Resonance' held at the joint EuroCMR-Society for Cardiovascular Magnetic Resonance (SCMR) meeting in Barcelona, Spain, in January 2018. The organizers of the meeting (P.G. and J.W.) appointed a scientific committee (A.J.S., L.Z., C.-J.C. and T.E.) to discuss the appropriateness of the meeting, clinical needs, topics to be discussed and, more importantly, to identify experts in the field to cover all aspects relevant to the goal of the meeting. The appointment of international experts was decided by general agreement among the members of the scientific committee. A follow-up meeting was arranged at the Joint Annual Meeting ISMRM-ESMRMB in Paris, France, in June 2018. A final teleconference was arranged in February 2019, after completion of an electronic database search and collection of evidence.

The evidence-based literature was synthesized by the following authors: P.G., A.J.S., L.Z. and C.-J.C. They searched electronic databases including MEDLINE 
(PubMed), Embase, the Cochrane library and OpenGray up to 8 January 2019, with no restriction on language. The search terms included "mitral regurgitation", "cardiovascular magnetic resonance" and "echocardiography". All retrospective and prospective studies that reported both CMR and echocardiography of MR were considered eligible. In addition, A.J.S. checked the reference lists of selected articles for further relevant articles. Review articles, case reports, comments and author replies were excluded. The final decision on inclusion was reached through a consensus of the four screening authors.

The main objectives at the meetings were to appraise previous and new lines of evidence on CMR-based assessment of the mitral valve, to review available data on the diagnostic and prognostic value of CMR in the MR setting, and to provide recommendations for the standardization of imaging protocols for use in clinical trials and experimental scenarios. For the consensus recommendations, open discussions took place between all experts, and verbal agreements were made. A majority of experts had to agree with a statement or recommendation for it to be included in this Consensus Statement. This Consensus Statement summarizes the final conclusions and recommendations agreed by the expert panel in the meetings.

\section{Current clinical guidelines}

The ESC and AHA/ACC guidelines for the management of valvular heart disease emphasize the severity of the MR in deciding whether patients are eligible for mitral valve surgery ${ }^{3,5}$, while also emphasizing the importance of assessing the haemodynamic effects of the MR on the left ventricle and left atrium. The AHA/ACC guidelines highlight that CMR is an appropriate test in chronic primary MR to assess ventricular volumes and function or even MR severity, especially when these issues are not satisfactorily addressed by transthoracic echocardiography $(\mathrm{TTE})^{5}$. In addition, for chronic secondary MR, CMR is indicated to establish and/or to assess myocardial viability, which in turn might influence the management of functional MR ${ }^{5}$. Similarly, the ESC guidelines on valvular heart disease recommend CMR assessment in patients with inadequate TTE imaging for ventricular volume and function assessment ${ }^{3}$. These guidelines do not detail several additional areas in which CMR can provide information on the aetiology of MR (primary or secondary),

\footnotetext{
Author addresses

${ }^{1}$ Department of Infection, Immunity and Cardiovascular Disease, University of Sheffield, Sheffield, UK.

${ }^{2}$ National Heart Centre Singapore, Duke-NUS Medical School, National University of Singapore, Singapore, Singapore.

${ }^{3}$ Department of Medical and Health Sciences, Linköping University, Linköping, Sweden. ${ }^{4}$ Department of Radiology, Leiden University Medical Center, Leiden, Netherlands. ${ }^{5}$ Department of Radiology, University of California-San Francisco, San Francisco, CA, USA.

${ }^{6}$ Bristol Heart Institute, Bristol National Institute of Health Research (NIHR) Biomedical Research Centre, University Hospitals Bristol NHS Trust and University of Bristol, Bristol, UK.

${ }^{7}$ Department of Cardiology, Leiden University Medical Center, Leiden, Netherlands. ${ }^{8}$ Departments of Cardiology and Cardiovascular Medicine, University of Oxford Centre for Clinical Magnetic Resonance Research, John Radcliffe Hospital, Oxford, UK.
}

including the assessment of mitral valve leaflet or scallop function ${ }^{6}$. Moreover, the guidelines have limited recommendations on how to perform comprehensive assessment of MR by CMR in a standardized way.

\section{Evidence for CMR to assess MR}

$\mathrm{CMR}$ is an emerging, noninvasive tool that can provide comprehensive assessment of the mitral valve and MR. As previously stated, CMR provides excellent accuracy and reproducibility in the assessment of ventricular and atrial size and function ${ }^{7}$, allowing for comprehensive longitudinal and postoperative assessment of reverse left ventricular (LV) remodelling. Studies evaluating the role of CMR for the assessment of MR are listed in TABLE 1. A thorough evaluation with the use of cine CMR allows a systematic inspection of the anatomy of the mitral valve and characterizes the MR, both of which contribute to determining the aetiology of the $\mathrm{MR}^{8}$. The severity of the MR can be evaluated using several CMR-based quantitative techniques that are detailed below. Furthermore, CMR can provide information about the mechanism of MR by identifying morphological abnormalities of the mitral valve apparatus ${ }^{9,10}$. The presence of billowing or flail segments can be identified by dedicated cine imaging focusing on the different scallops of the mitral valve leaflets ${ }^{8}$. In secondary MR, CMR can provide an accurate assessment of LV dilatation and (dys)function, in addition to the identification of myocardial and papillary muscle scar formation ${ }^{11}$.

\section{Clinical outcome studies}

Primary MR. In a prospective, multicentre study by Myerson and colleagues, 109 asymptomatic patients with moderate or severe primary MR defined by echocardiography had CMR scans at baseline and were followed up for up to 8 years (mean $2.5 \pm 1.9$ years ${ }^{12}$. CMR quantification of MR accurately identified patients who progressed to having symptoms or other indications for surgery: $91 \%$ of participants with a regurgitant volume $\leq 55 \mathrm{ml}$ survived to 5 years without surgery compared with only $21 \%$ of participants with a regurgitant volume $>55 \mathrm{ml}(P<0.0001)^{12}$. Similar findings were observed in a further prospective, dual-centre study by Penicka and colleagues in which 258 asymptomatic patients with moderate or severe primary MR defined by echocardiography underwent CMR and were followed up for a median of 5 years ${ }^{13}$. In this study, the researchers demonstrated that CMR-derived MR volume was the best predictor of mortality (area under the curve $=0.72$ ). In addition, when MR volume was combined with the development of an indication for mitral valve surgery as a clinical outcome, the predictive value increased (area under the curve $=0.83)^{13}$. The other major finding of this study was that the agreement between echocardiography and CMR for classifying primary MR was poor for patients with late

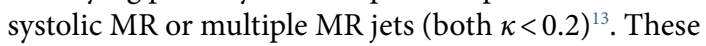
findings suggest that, in patients who have complex primary MR jet physiology, standard CMR quantification of MR can offer complementary information to that obtained by echocardiography for consideration of valvular intervention. 


\section{CONSENSUS STATEMENT}

\begin{tabular}{|c|c|c|c|c|}
\hline Study (year) & $n$ & Prospective study? & Correlation $(r)^{\mathrm{a}}$ & Bias $(m l)^{b}$ \\
\hline \multicolumn{5}{|l|}{ LVSV-AoPC method } \\
\hline Penicka et al. $(2018)^{13}$ & 258 & Yes & 0.61 & $17.1 \pm 28.9$ \\
\hline \multirow[t]{3}{*}{ Heo et al. $(2017)^{44}$} & \multirow[t]{3}{*}{37} & \multirow[t]{3}{*}{ Yes } & PISA: 0.81 & $-15.2 \pm 18.3$ \\
\hline & & & 2D volumetric: 0.56 & $-17.4 \pm 29.4$ \\
\hline & & & 3D echo: 0.94 & $8.7 \pm 11.6$ \\
\hline Harris et al. $(2017)^{45}$ & 22 & Yes & - & - \\
\hline Sachdev et al. $(2017)^{46}$ & 50 & Yes & 0.79 & $-0.6(-43$ to 44$)$ \\
\hline Myerson et al. $(2016)^{12}$ & 109 & Yes & - & - \\
\hline Aplin et al. $(2016)^{47}$ & 72 & Yes & 0.80 & $11 \pm 28$ \\
\hline Lopez-Mattei et al. $(2016)^{48}$ & 70 & No & 0.59 & $2 \pm 17$ \\
\hline Uretsky et al. $(2015)^{19}$ & 103 & Yes & 0.60 & $16(-38$ to 70$)$ \\
\hline \multirow[t]{2}{*}{ Brugger et al. $(2015)^{49}$} & \multirow[t]{2}{*}{55} & \multirow[t]{2}{*}{ Yes } & 3D TOE PISA: 0.87 & $-5.9(-26.5$ to 14.7$)$ \\
\hline & & & 3D TTE PISA: 0.74 & $-11.8(-39.4$ to 15.8$)$ \\
\hline \multirow[t]{2}{*}{ Choi et al. (2014) } & \multirow[t]{2}{*}{52} & \multirow[t]{2}{*}{ Yes } & 2D TTE PISA: 0.84 & $-10.4(-29.8$ to 8.9$)$ \\
\hline & & & 3D TTE PISA: 0.91 & $0.9(-12.8$ to 14.7$)$ \\
\hline \multirow[t]{2}{*}{ Van De Heyning et al. $(2013)^{51}$} & \multirow[t]{2}{*}{38} & \multirow[t]{2}{*}{ Yes } & 2D TTE Doppler:-0.14 & 39 (limits not reported) \\
\hline & & & TTE PISA: 0.45 & 30 (limits not reported) \\
\hline \multirow{2}{*}{$\begin{array}{l}\text { Thavendiranathan et al. } \\
(2013)^{52}\end{array}$} & \multirow[t]{2}{*}{35} & \multirow[t]{2}{*}{ Yes } & 3D integrated PISA: 0.92 & $1.4(-17$ to 19.8$)$ \\
\hline & & & 3D peak PISA: 0.87 & $15.3(-10.2$ to 40.8$)$ \\
\hline \multirow[t]{3}{*}{ Son et al. $(2013)^{53}$} & \multirow[t]{3}{*}{32} & \multirow[t]{3}{*}{ Yes } & 2D PISA: 0.55 & $7.9(-46.9$ to 62.8$)$ \\
\hline & & & 2D VM: 0.58 & $16.7(-44.9$ to 78.2$)$ \\
\hline & & & 3D FVCD: 0.85 & $5.7(-27.9$ to 39.3$)$ \\
\hline Reddy et al. $(2013)^{54}$ & 44 & Yes & - & - \\
\hline \multirow[t]{2}{*}{ Cawley et al. $(2013)^{55}$} & \multirow[t]{2}{*}{10} & \multirow[t]{2}{*}{ Yes } & PISA: 0.96 & $-4(-29$ to 22$)$ \\
\hline & & & Doppler: 0.85 & 21 (-28 to 72$)$ \\
\hline \multirow[t]{2}{*}{ Hamada et al. $(2012)^{56}$} & \multirow[t]{2}{*}{46} & \multirow[t]{2}{*}{ Yes } & EROA: 0.75 & 20 (-41 to 81$)$ \\
\hline & & & AROA:- & 13 (-22 to 47$)$ \\
\hline Skaug et al. $(2010)^{57}$ & 27 & Yes & 0.78 & $-4.7 \pm 30.6$ \\
\hline Shanks et al. $(2010)^{43}$ & 30 & Yes & 2DTTE:- & -12.4 (-45.6 to 20.8$)$ \\
\hline & & & 3D TEE:- & $-2.32(-18.6$ to 13.9$)$ \\
\hline Myerson et al. $(2010)^{58}$ & 55 & Yes & - & - \\
\hline Hellgren et al. (2008) & 18 & Yes & - & $-27.5(-65.4$ to 10.3$)$ \\
\hline Gabriel et al. $(2008)^{60}$ & 27 & Yes & - & - \\
\hline Gelfand et al. $(2006)^{61}$ & 107 & Yes & - & - \\
\hline Kizilbash et al. (1998) ${ }^{62}$ & 22 & Yes & 0.92 & $3 \pm 13$ \\
\hline Hundley et al. $(1995)^{63}$ & 17 & Yes & - & - \\
\hline LVSV-RVSV method & & & & \\
\hline Sukpraphrute et al. (2012) & 43 & No & PISA: 0.48 & $-6.4(-49$ to 36$)$ \\
\hline Kon et al. $(2004)^{35}$ & 28 & No & - & - \\
\hline MVPC-AoPC method & & & & \\
\hline Polte et al. $(2017)^{64}$ & 40 & Yes & - & - \\
\hline Buck et al. $(2008)^{65}$ & 73 & Yes & 0.63 & $-13.5 \pm 10.3$ \\
\hline Fujita et al. (1994) & 19 & Yes & - & - \\
\hline
\end{tabular}


Table 1 | (cont.) Studies assessing the use of CMR in MR with or without echocardiography

\begin{tabular}{|c|c|c|c|c|}
\hline Study (year) & $n$ & Prospective study? & Correlation $(r)^{\mathrm{a}}$ & $\operatorname{Bias}(\mathrm{ml})^{\mathrm{b}}$ \\
\hline \multicolumn{5}{|l|}{ 4D-flow methods } \\
\hline Kamphuis et al. (2019) ${ }^{67}$ & 160 & No & - & - \\
\hline \multirow[t]{2}{*}{ Feneis et al. $(2018)^{30}$} & \multirow[t]{2}{*}{21} & \multirow[t]{2}{*}{ No } & Direct: 0.81 & - \\
\hline & & & Indirect: 0.97 & - \\
\hline Calkoen et al. $(2015)^{37}$ & 32 & Yes & $0.50-0.60$ & - \\
\hline Roes et al. $(2009)^{28}$ & 51 & No & - & - \\
\hline \multirow[t]{2}{*}{ Marsan et al. $(2009)^{68}$} & \multirow[t]{2}{*}{64} & \multirow[t]{2}{*}{ Yes } & 3D TTE: 0.94 & $-0.08(-7.7$ to 7.6$)$ \\
\hline & & & 2DTTE:- & $-2.9(-18$ to 12.5$)$ \\
\hline Westenberg et al. $(2008)^{38}$ & 30 & No & - & - \\
\hline \multicolumn{5}{|l|}{ Other quantitative methods } \\
\hline Gorodisky et al. (2018) ${ }^{69}$ & 27 & Yes & $\begin{array}{l}\text { CMR PISA versus echo } \\
\text { PISA: } 0.87\end{array}$ & - \\
\hline Uretsky et al. $(2010)^{70}$ & 23 & No & - & - \\
\hline \multicolumn{5}{|l|}{ Nonquantitative methods } \\
\hline Heitner et al. $(2012)^{71}$ & 68 & No & 0.47 & - \\
\hline Ozdogan et al. (2009) & 21 & No & - & - \\
\hline Buchner et al. $(2008)^{34}$ & 35 & Yes & $\begin{array}{l}\text { CMR EROA versus echo } \\
\text { EROA: } 0.81\end{array}$ & - \\
\hline Aurigemma et al. $(1990)^{73}$ & 50 & Yes & - & - \\
\hline Pflugfelder et al. (1989) & 26 & Yes & - & - \\
\hline \multicolumn{5}{|c|}{$\begin{array}{l}\text { AoPC, aortic phase-contrast stroke volume; AROA, anatomical regurgitant orifice area; CMR, cardiac magnetic resonance } \\
\text { imaging; echo, echocardiography; EROA, effective regurgitant orifice area; FVCD, full-volume colour Doppler echocardiography; } \\
\text { LVSV, left ventricular stroke volume; MR, mitral regurgitation; MVPC, mitral valve phase-contrast stroke volume; PISA, proximal } \\
\text { isovelocity surface area; RVSV, right ventricular stroke volume; TOE, transoesophageal echocardiography; TTE, transthoracic } \\
\text { echocardiography; VM, volumetric quantification method. Between echocardiography-determined and CMR-determined MR } \\
\text { volume. 'Regurgitant volume (echocardiography - CMR). }\end{array}$} \\
\hline
\end{tabular}

LGE has been reported on CMR images in patients with primary MR, especially in those with mitral valve prolapse $^{8,14}$. In addition, in patients with primary MR, LGE of papillary muscles is associated with complex ventricular arrhythmias ${ }^{15}$. Subsequent evidence confirms that LV fibrosis indicated by LGE is more prevalent in MR with mitral valve prolapse than in patients without prolapse, whereas patients with mitral valve prolapse and concomitant LV fibrosis have the highest rate of arrhythmic events ${ }^{15}$. Persistent volume overload from MR results in impaired LV function and the presence of diffuse myocardial fibrosis ${ }^{16}$. Mitral annulus disjunction is an abnormal atrial displacement of the hinge point of the mitral valve away from the ventricular myocardium $^{17}$. Mitral annulus disjunction has been associated with mitral valve prolapse and sudden cardiac death owing to ventricular arrhythmias ${ }^{9,18}$.

Secondary MR. A prospective, multicentre study in 103 patients with either primary or secondary MR showed substantial discordance in the severity of MR as assessed with either CMR or echocardiography on the basis of either the American Society of Echocardiography integrated method or the proximal isovelocity surface area (PISA)-based regurgitant volume ${ }^{19}$. In addition, in the subset of patients who had mitral valve surgery and underwent postoperative CMR, good correlation existed between LV remodelling and MR severity as assessed by CMR $(r=0.85 ; P<0.0001)$, but not when assessed by echocardiography $(r=0.32 ; P=0.1)$, either categorically or quantitatively, with the use of the PISA method.

Persistent volume overload from MR results in impaired LV function and subsequent myocardial fibrosis ${ }^{16}$. In patients with ischaemic cardiomyopathy and severe $M R$, the presence of severe scarring in the region of the posterior papillary muscle, as detected by preoperative CMR, can render these patients unsuitable for mitral annuloplasty ${ }^{20}$. Moreover, the extent of myocardial scarring is also informative about the progression of ischaemic $\mathrm{MR}^{21}$.

In summary, CMR has become an established noninvasive imaging modality to assess the severity of MR. CMR can be used to phenotype prognostically relevant clinical features that are complementary to those identified by echocardiography in patients with MR.

\section{CMR acquisition protocol to assess MR}

A CMR study should aim to answer several clinical questions (BOX 1) that influence the management of patients with MR. A comprehensive MR protocol should assess the mitral valve anatomy and function to define the cause of the MR - primary (organic) versus secondary (functional), LV and right ventricular (RV) volumes and function, and quantify the MR (FIG. 1a). However, given that TTE, the first-line imaging test, can provide information on the aetiology of the MR in the majority of patients, we also include a CMR protocol focused on MR quantification (FIG. 1 b). A decision on whether to use 


\section{Box 1 | Clinical questions in CMR assessment of MR \\ A comprehensive cardiovascular magnetic resonance imaging (CMR) study should aim to answer the following clinical questions in the assessment of mitral regurgitation (MR) for consideration of mitral valvular intervention ${ }^{3}$. \\ What is the aetiology of the MR? \\ - Primary or secondary \\ - Presence and location of myocardial infarction on late gadolinium enhancement imaging \\ How severe is the MR? \\ Are any signs present on imaging in asymptomatic patients that might indicate worse outcome if valve intervention is delayed? \\ - Dilated left ventricle \\ - Borderline reduced left ventricular ejection fraction \\ - Dilated left atrium \\ - Progressive dilatation of the left ventricle and worsening of left ventricular function \\ Has the MR worsened? \\ - On longitudinal CMR studies, has the MR volume or MR fraction worsened?}

the comprehensive or the focused CMR protocol should be made depending on the quality of the information gained from TTE.

\section{Cine images}

Standard cine CMR should be performed according to the SCMR recommendations ${ }^{22}$ :

- Standard, long-axis, steady state, free-precession cine images: four-chamber (horizontal long-axis), two-chamber (vertical long-axis) and three-chamber (LV outflow tract view).

- A stack of contiguous cines perpendicular to the mitral commissures, transecting the principal line of coaptation, approximately in a modified LV outflow tract plane. These should have a slice thickness of $5 \mathrm{~mm}$ and no gap, with a temporal resolution of $\geq 45 \mathrm{~ms}$ (REF. ${ }^{23}$ ) (FIG. 2). The main aim is to cover all the mitral scallops: A1-P1, A2-P2 and A3-P3. Additional commissural cines are acquired perpendicular to the lines of coaptation next to each of the commissures if the commissures are at an oblique angle to the central coaptation line (FIG. 2, lines 'a' and 'b').

- Standard, contiguous, short-axis, LV cine stack with extended coverage of the mitral valve.

- Specific short-axis cine perpendicular to the tips of the mitral valve in systole (if an optimal image is not obtained within the LV stack).

Tips. On cine acquisitions, flow turbulence (for example, because of MR jets) produces spin-spin dephasing, which can be visualized as hypointense areas within the blood pool inside the relevant cardiac chamber. This phenomenon allows the observer to make a gross qualitative assessment of the MR. If steady state, freeprecession cines do not show the flow void clearly, fast spoiled gradient-recalled echo cines with longer repetition and echo times can be performed to visualize the MR regurgitant flow void better. However, this qualitative MR assessment is very susceptible to changes in cine pulse sequences and therefore should be used cautiously. Visualization of the MR jet on cine images can, however, inform about the aetiology (in addition to cine imaging of leaflet anatomy and function) - eccentric jets associated with mitral valve leaflet prolapse versus a central jet associated with mitral annular dilatation or LV dilatation resulting in non-coaptation. Aetiologies of MR that are more challenging to diagnose, such as cleft mitral valve or perforated leaflets, can also be assessed well with a combination of cine and flow imaging. Owing to variations in breath holds, a stack of contiguous 5-mm slices can have spatial misalignment, which should be carefully judged during scanning. If the cusp views are missed during scanning, additional imaging can be required.

\section{Flow imaging}

$2 D$ phase-contrast flow. Currently, the standard approach to flow imaging is $2 \mathrm{D}$ phase-contrast, velocityencoded imaging. For this approach, one-direction (through-plane), motion-encoded, cine gradient-echo sequences are used. The preferred sequence parameters for $2 \mathrm{D}$ phase-contrast imaging are included in the SCMR recommendations ${ }^{22}$ :

- Aortic forward flow: a through-plane image plane should be placed at the sino-tubular junction in end diastole to quantify forward flow for the calculation of MR volume (LV stroke volume minus aortic forward flow). This plane should be perpendicular to the vessel. In published studies, baseline velocity encoding for aortic flow is $2.0-2.5 \mathrm{~m} / \mathrm{s}$. If there is significant aliasing, consider increasing the velocity encoding or imaging slightly higher than the previously prescribed phase-contrast through-plane.

- MR visual assessment: a long-axis LV outflow track phase-contrast stack that is perpendicular to the commissures, aligned with the direction of inflow and transecting the principal line of coaptation, is recommended to visualize MR jets in multiple planes. This visualization will clarify the aetiology of the MR. Alternatively, an experienced operator can plan a single image to capture through-plane flow on the atrial side of the valve.

Tips. Signal averaging can be used within the limits of breath-holding capabilities. Free breathing, respiratory navigator-based signal-averaging techniques can be applied to improve the temporal or spatial resolution if necessary. The potential for background flow offset errors can be reduced by ensuring that phase-contrast sequences are acquired with the region of interest (the ascending aorta) located at the iso-centre of the magnet to minimize any inhomogeneities in the magnetic field ${ }^{24}$. Background phase offset errors can significantly hinder the accuracy of flow measurement ${ }^{25}$, and background flow correction processes should be used, such as the interpolated automatic sequence ${ }^{26}$, where available. In patients with clinically significant aortic sinus turbulent flow, the through-plane image plane can be positioned at 


\section{CONSENSUS STATEMENT}

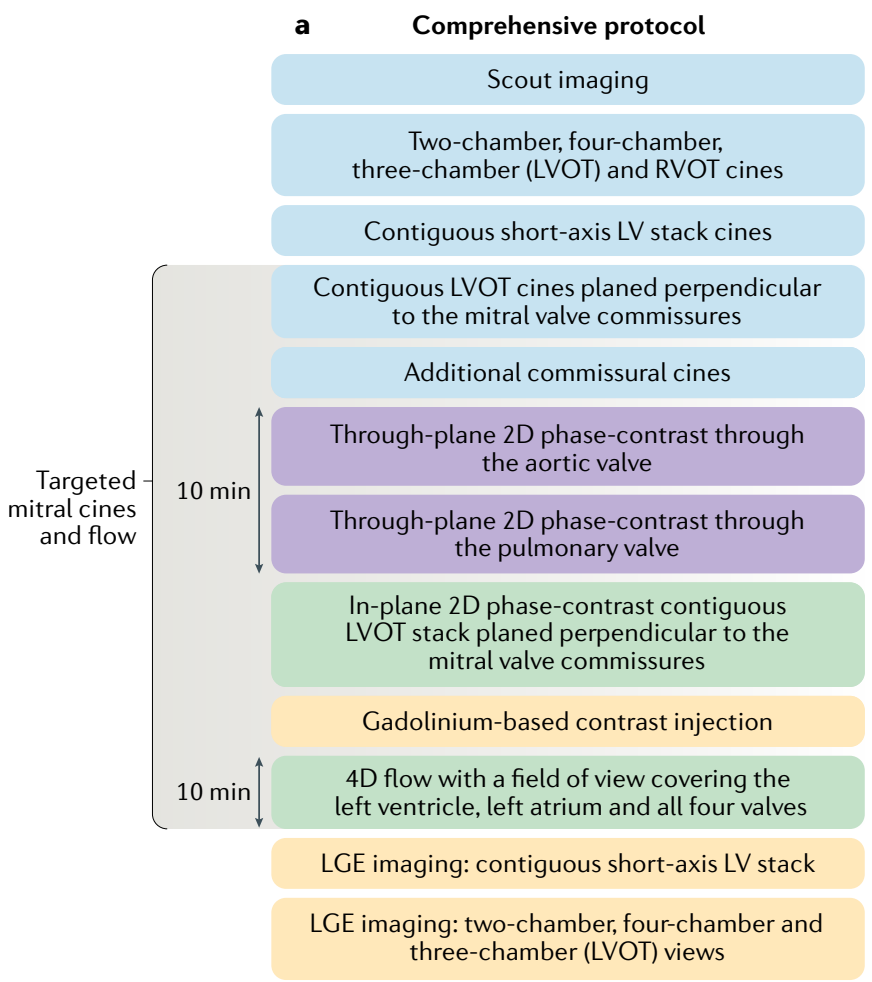

b Focused, quantitative protocol

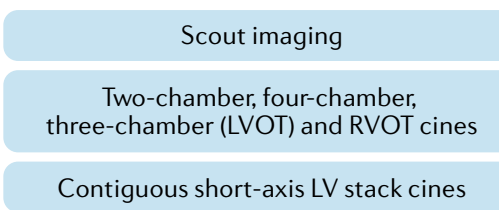

Through-plane 2D phase-contrast through the aortic valve

Through-plane 2D phase-contrast through the pulmonary valve

$4 \mathrm{D}$ flow with a field of view covering the left ventricle, left atrium and all four valves

Fig. 1 | Recommended cardiovascular magnetic resonance imaging protocols for the assessment of mitral regurgitation. a | Comprehensive cardiovascular magnetic resonance imaging protocol for the assessment of mitral regurgitation. b | Focused, quantitative protocol. LGE, late gadolinium enhancement; LV, left ventricular; LVOT, left ventricular outflow tract; RVOT, right ventricular outflow tract.

the level of the main pulmonary artery in the ascending aorta to quantify aortic forward flow.

In patients with arrhythmias (mostly atrial fibrillation), consider performing multiple phase-contrast acquisitions and also using arrhythmia-rejection sequences. If arrhythmia rejection is used for phasecontrast acquisitions, it should be similarly applied to the functional cine images. This approach will at least provide consistency between the flow and the functional measurements. Performing 2D phase-contrast through the mitral valve for forward and backward flow quantification is not recommended, mainly because this technique remains highly susceptible to through-plane mitral annular motion. Furthermore, for dynamic regurgitant jets, the acquisition plane cannot be adapted to the changing direction of flow.

$4 D$ phase-contrast flow. The $2 \mathrm{D}$ phase-contrast can be swapped for $4 \mathrm{D}$ phase-contrast flow if local established expertise and technical knowledge exist to quantify transvalvular flow with this approach. $4 \mathrm{D}$-flow CMR techniques offer further improvements in the assessment of MR and are entering clinical practice ${ }^{27}$. Advantages of MR quantification with the use of $4 \mathrm{D}$-flow CMR include single-acquisition, single-sequence, retrospective analysis that allows valve tracking to account for motion throughout the cardiac cycle as well as direct measurement of $\mathrm{MR}^{28}$. Direct quantification of the regurgitant jet is particularly useful in pathologies involving multiple valves. A systematic review of
4D flow-derived methods for MR quantification identified seven studies that demonstrated that $4 \mathrm{D}$ flowderived MR volume is similar to that derived using standard CMR methods and even to that derived using 3D transoesophageal echocardiography (TOE) methods ${ }^{29}$. In one study, a standard CMR method for quantification of $\mathrm{MR}$ volume and $4 \mathrm{D}$ flow-derived methods yielded similar results ${ }^{30}$.

For 4D-flow CMR, a retrospectively electrocardiogramgated sequence covering the complete cardiac cycle, with a temporal resolution of $\geq 45 \mathrm{~ms}$ and a spatial resolution of $3 \mathrm{~mm} \times 3 \mathrm{~mm} \times 3 \mathrm{~mm}$ or higher is recommended ${ }^{31}$. The field of view should preferably cover the whole left ventricle, left atrium and aortic outflow track, including the proximal ascending aorta. Before analysis, $4 \mathrm{D}$ velocity data should be carefully checked for errors and, where possible, these errors should be resolved.

\section{LGE imaging}

LGE imaging should be performed in accordance with published guidelines ${ }^{22}$. Contiguous, short-axis, LV stack LGE imaging is recommended, in addition to LGE in the three standard long-axis planes.

\section{Analysis \\ Mitral valve anatomy}

Leaflet morphology. A visual assessment should be made of all four components of the mitral valve: the anterior and posterior leaflets, the annulus, the subvalvular apparatus (papillary muscles), and LV contractility (including 


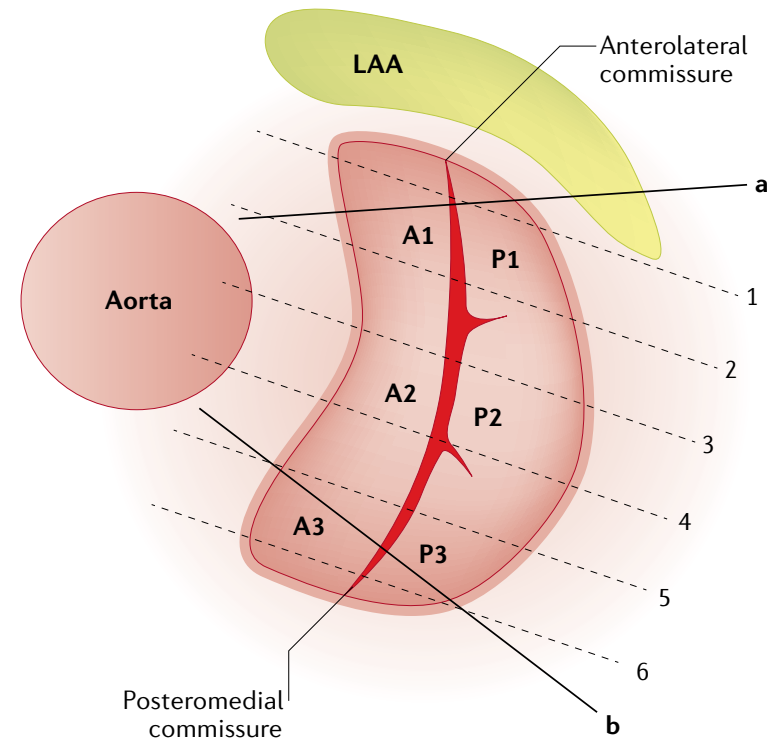

Medial $\underset{\text { Anterior }}{\stackrel{\uparrow}{\longrightarrow} \text { Lateral }}$

Fig. 2 | Mitral valve assessment planing during cine cardiovascular magnetic resonance imaging acquisition. A contiguous, long-axis, left ventricular outflow tract cine cardiovascular magnetic resonance imaging stack should be acquired to visualize and assess all the mitral valve cusps (A1-P1, A2-P2 and A3-P3). Extra planes to the commissural line also need to be considered, as demonstrated by the lines ' $a$ ' and ' $b$ '. LAA, left atrial appendage. cines and is defined as being clinically significant if the distance is $\geq 1.0 \mathrm{~mm}$.

Leaflet motion. Leaflet motion can be described using Carpentier's classification: type I (normal leaflet motion); type II (excessive leaflet motion); and type III (restricted leaflet motion), subcategorized as type IIIa (restricted during both systole and diastole) and type IIIb (restricted only during systole). After a comprehensive review of leaflet morphology and motion, a possible aetiology for the MR should be described according to TABLE 2. Case studies are provided in FIG. 3. The aetiology should be consistent with the overarching diagnosis. Sometimes, a mixed picture of both primary and secondary MR can exist - for example, pre-existing secondary MR caused by dilated cardiomyopathy together with a newly torn chord or flail leaflet.

\section{Methods of MR quantification}

Several qualitative and quantitative methods of MR assessment by CMR are available.

Qualitative assessment. The MR jet should be visualized using both cine and 2D phase-contrast CMR, as described in the MR assessment protocol. This approach is mainly performed by visual assessment of the MR jet on the basis of spin dephasing on cine images. MR jet characterization should include whether the jet is central or eccentric, and early, mid, late or pan systolic.

4D-flow CMR allows for visualization of $2 \mathrm{D}$ velocity vectors in any plane, facilitating a comprehensive assessment of the blood flow dynamics in the left atrium ${ }^{32,33}$. $M R$ jets are dynamic and can change directions during systole depending on mitral leaflet adaptations. Velocity vector visualization of LA flow coupled with cine CMR can help to understand the cause of the MR (FIG. 4). Velocity vector visualization of the velocity jet is preferred over spin dephasing because it provides truly quantitative, directional velocity data. In addition, this method can offer a better assessment of the extent of MR than is provided by Doppler imaging (which is single velocity-encoded imaging), especially when the MR jet is swirling within the left atrium.

\section{Table 2 | Modified Carpentier's classification of mitral valve morphology and MR aetiology}

\begin{tabular}{|c|c|c|c|c|}
\hline \multirow[t]{2}{*}{ Type of leaflet motion } & \multicolumn{2}{|c|}{ Normal mitral valve leaflet } & \multicolumn{2}{|l|}{ Abnormal mitral valve leaflet } \\
\hline & $\begin{array}{l}\text { Leaflet lesion } \\
\text { (morphology) }\end{array}$ & Aetiology: secondary MR & Leaflet lesion (morphology) & Aetiology: primary MR \\
\hline $\begin{array}{l}\text { Type I: normal leaflet } \\
\text { motion }\end{array}$ & Annular dilatation & $\begin{array}{l}\text { Dilated cardiomyopathy }{ }^{\mathrm{a}} \text { or } \\
\text { left atrial dilatation }\end{array}$ & Leaflet perforation (tear) & Endocarditis \\
\hline $\begin{array}{l}\text { Type II: excess leaflet } \\
\text { motion (prolapse or flail) }\end{array}$ & - & - & $\begin{array}{l}\text { Elongation (rupture of } \\
\text { chordae or papillary muscle) }\end{array}$ & $\begin{array}{l}\text { Degenerative valve disease, } \\
\text { endocarditis, trauma or } \\
\text { ischaemic cardiomyopathy }\end{array}$ \\
\hline $\begin{array}{l}\text { Type Illb: restricted } \\
\text { leaflet motion (mainly } \\
\text { in systole) }\end{array}$ & $\begin{array}{l}\text { Left ventricular } \\
\text { dilatation (aneurysm) }\end{array}$ & $\begin{array}{l}\text { Ischaemic cardiomyopathy }{ }^{a} \\
\text { or dilated cardiomyopathy }\end{array}$ & $\begin{array}{l}\text { Papillary muscle displacement } \\
\text { or chordae tethering }\end{array}$ & $\begin{array}{l}\text { Ischaemic cardiomyopathy } \\
\text { or dilated cardiomyopathy }\end{array}$ \\
\hline
\end{tabular}

MR, mitral regurgitation. ${ }^{a}$ Mixed aetiology. 
Quantitative assessment. CMR planimetry of the anatomical mitral regurgitant lesion in patients with $\mathrm{MR}$ is feasible and allows quantification of $\mathrm{MR}$, which has been shown to have good agreement with quantification by other invasive and noninvasive methods ${ }^{34}$. Quantification of mitral regurgitant volume and fraction is the recommended technique because most clinical outcome data are available. The MR volume can be obtained by four different methods (FIG. 5).

1. The difference between the LV stroke volume (LVSV) calculated using planimetry of cine steady

a Primary organic MR

Morphology
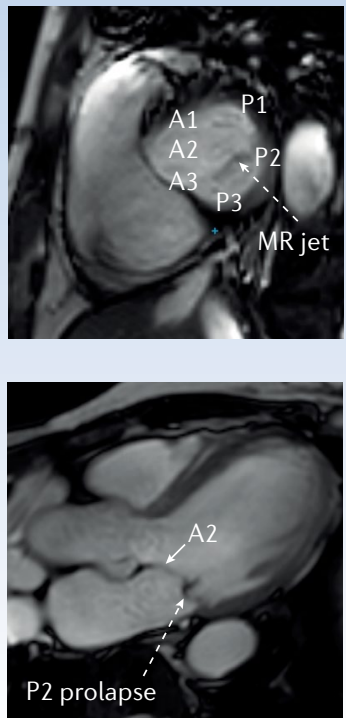

b Secondary functional MR Morphology
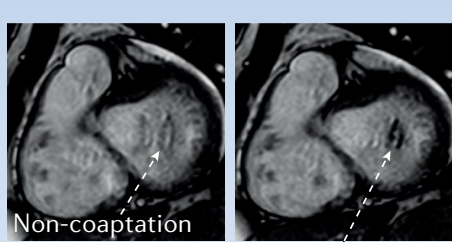

Central MR jet
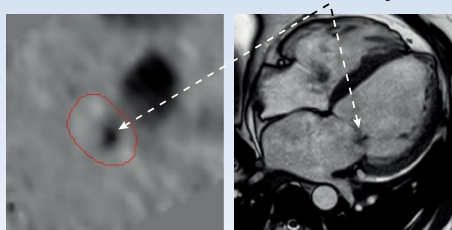

Extensive transmural scar in the anterior and inferior left ventricular walls

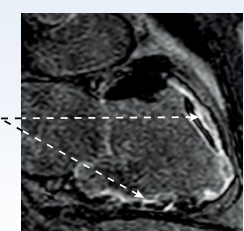

MR quantification
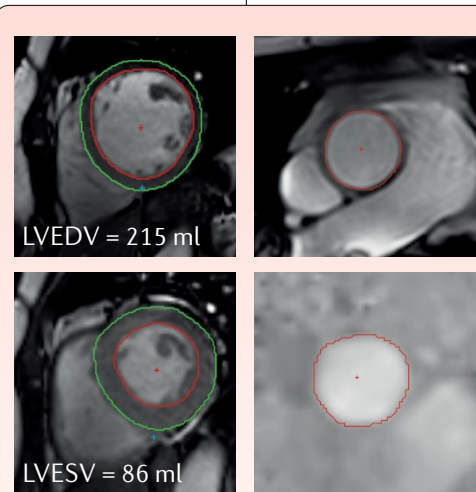

$\mathrm{LVSV}=\mathrm{LVEDV}-\mathrm{LVESV}=129 \mathrm{ml}$

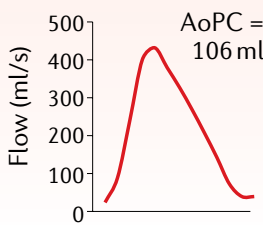

MR quantification
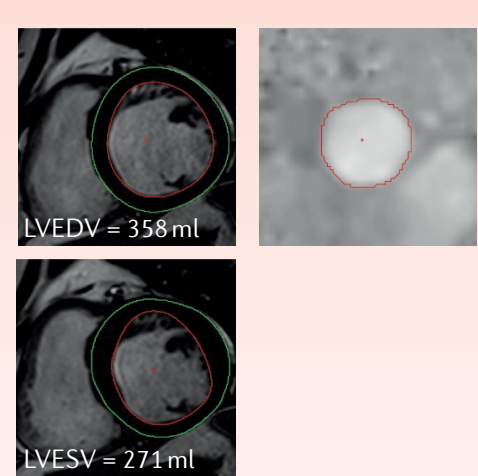

LVSV $=$ LVEDV - LVESV $=87 \mathrm{ml}$

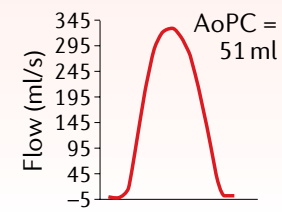

Diagnosis

Aetiology
- Primary MR
- Carpentier type II
- P2 prolapse

MR severity

- $M R=L V S V-A o P C=23 \mathrm{ml}$

- $\mathrm{MR}_{\mathrm{RF}}^{\mathrm{vol}}=\left(\mathrm{MR}_{\mathrm{vol}} / \mathrm{LVSV}\right) \times 100=18 \%$

Overall

Mild MR

Fig. 3 | Case studies of primary and secondary MR. a | Mitral regurgitation (MR) assessment with cardiovascular magnetic resonance imaging in a patient with organic MR. Prolapse of the P2 can be seen on the three-chamber view during midsystole (morphology panel, bottom image), and the resulting MR jet is visualized on the short-axis view (morphology panel, top image). The MR volume (MR $\mathrm{vol}_{\text {) }}$ is quantified using the standard method: left ventricular stroke volume (LVSV) minus aortic phase-contrast forward volume (AoPC). b | MR assessment in a patient with ischaemic cardiomyopathy. Noncoaptation owing to ventricular dilatation is seen on the short-axis cines (morphology panel, top images). A through-plane phase-contrast acquisition shows the central MR jet (morphology panel, right-hand middle image). Late gadolinium enhancement imaging reveals extensive ischaemic myocardial scaring (Morphology panel, right-hand bottom image). LVEDV, left ventricular end-diastolic volume; LVESV, left ventricular end-systolic volume; $\mathrm{MR}_{\mathrm{RF}}$, mitral regurgitation fraction. 
End diastole
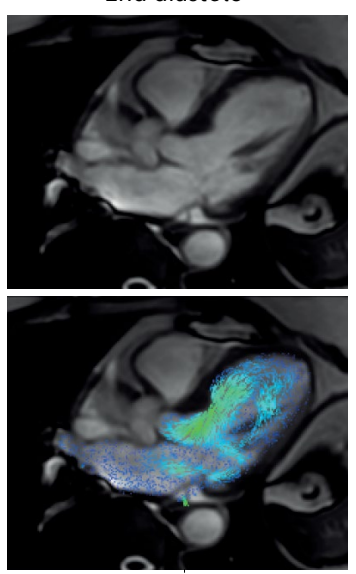

Early systole
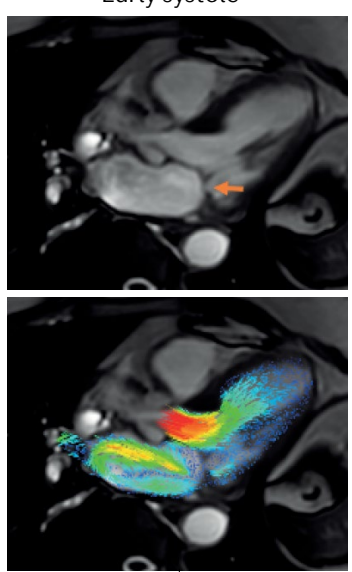

Mid systole
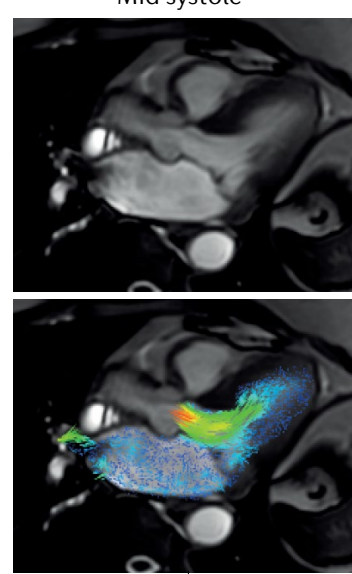
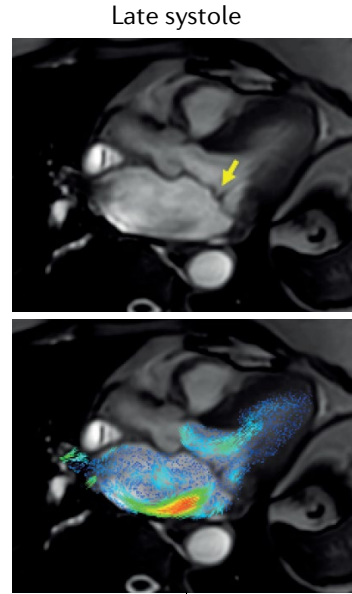

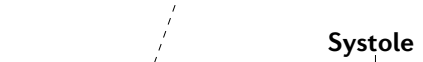

Diastole

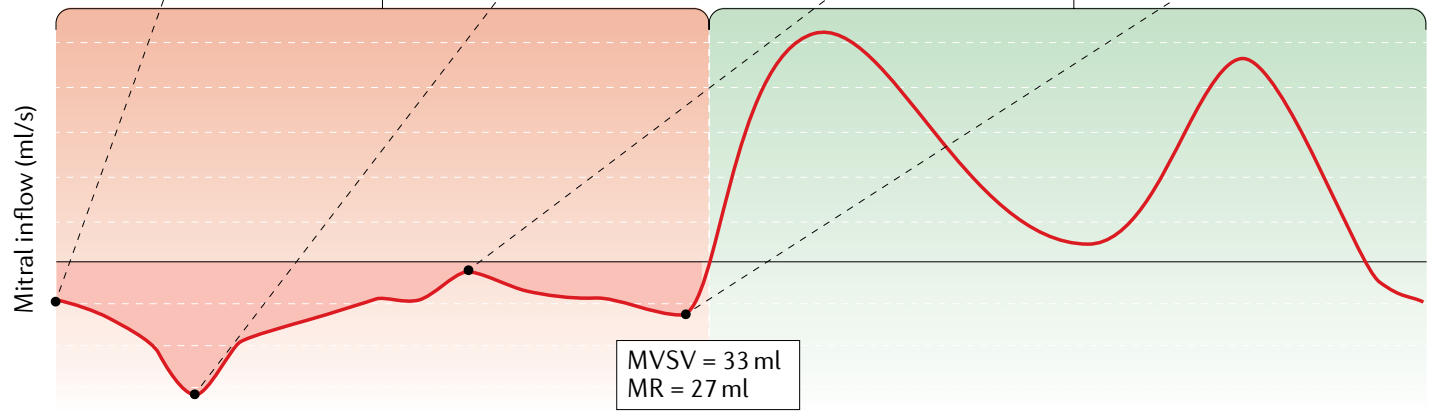

Fig. 4 | Time-resolved velocity vector visualization with CMR of dynamically changing MR jet. The top row shows cardiovascular magnetic resonance imaging (CMR) of three-chamber cines acquired during four phases of the cardiac cycle: end diastole, early systole, mid systole and late systole. The second row of images shows velocity vectors in the left atrium and the left ventricle superimposed on the three-chamber cines. These images allow the visualization of the A2-P2 scallops, demonstrating early systolic prolapse of the P2 scallop (orange arrow) resulting in a mitral regurgitation (MR) jet directed towards the medial interatrial septum, which settles in mid systole. A late-systolic, posteriorly directed MR jet (yellow arrow) can be appreciated as a result of A2 prolapse. The MR volume is quantified in the lower panel. This example highlights how cine CMR and augmented streamline visualization of the 4D-flow CMR can help to make a more dynamic pathophysiological diagnosis of the cause of MR. MVSV, mitral valve stroke volume.

state, free-precession images and the aortic (systolic) forward volume obtained by phase-contrast images (AoPC); the standard approach.

2. The difference between the LVSV and the RV stroke volume (RVSV) calculated using planimetry of cine steady state, free-precession images; this approach assumes no other valve regurgitation or haemodynamically significant shunt.

3. The difference between the mitral inflow stroke volume and the AoPC.

4. Direct quantification of MR flow by $4 \mathrm{D}$-flow $\mathrm{CMR}$, with retrospective mitral valve tracking.

Among these methods, the first is the most widely used and has the most prognostic data available ${ }^{12,13}$. This method allows the quantification of the regurgitant volume without considering regurgitant jet morphology. This approach is particularly helpful in patients with multiple or eccentric jets, or variable jets through systole. In addition, this approach is independent of the effects of aortic, tricuspid and pulmonary regurgitation. However, this method requires a combination of two acquisitions and is, therefore, subject to potential interscan variability.
If any issues exist with the acquired AoPC to quantify MR by the LVSV - AoPC method, an alternative approach is to use the pulmonary artery flow (PaPC), as long as no intracardiac shunts exist (LVSV - PaPC). Moreover, the $\mathrm{PaPC}$ approach might be advantageous in certain circumstances because the pulmonary valve is less often diseased and therefore less susceptible to creating errors from turbulent flow. In patients with a restrictive ventricular septal defect and a unidirectional left-to-right shunt, the LVSV - PaPC method can be used to quantify MR. In patients with a bidirectional flow ventricular septal defect, this method is not applicable, and direct measurement of the MR jet should be considered. In patients with an atrial septal defect, the standard method (LVSV - AoPC) is still appropriate for quantification of $\mathrm{MR}$ and also allows the shunt to be assessed using the ratio of the pulmonary and aortic flows (PaPC:AoPC).

The difference in LVSV and RVSV can also be used to quantify $\mathrm{MR}^{31}$. However, given the relatively lower precision with which RVSV is quantified compared with LVSV, substantial bias in MR volume can be introduced between two operators, resulting in reduced reliability ${ }^{35}$. In addition, this method is not valid for patients 


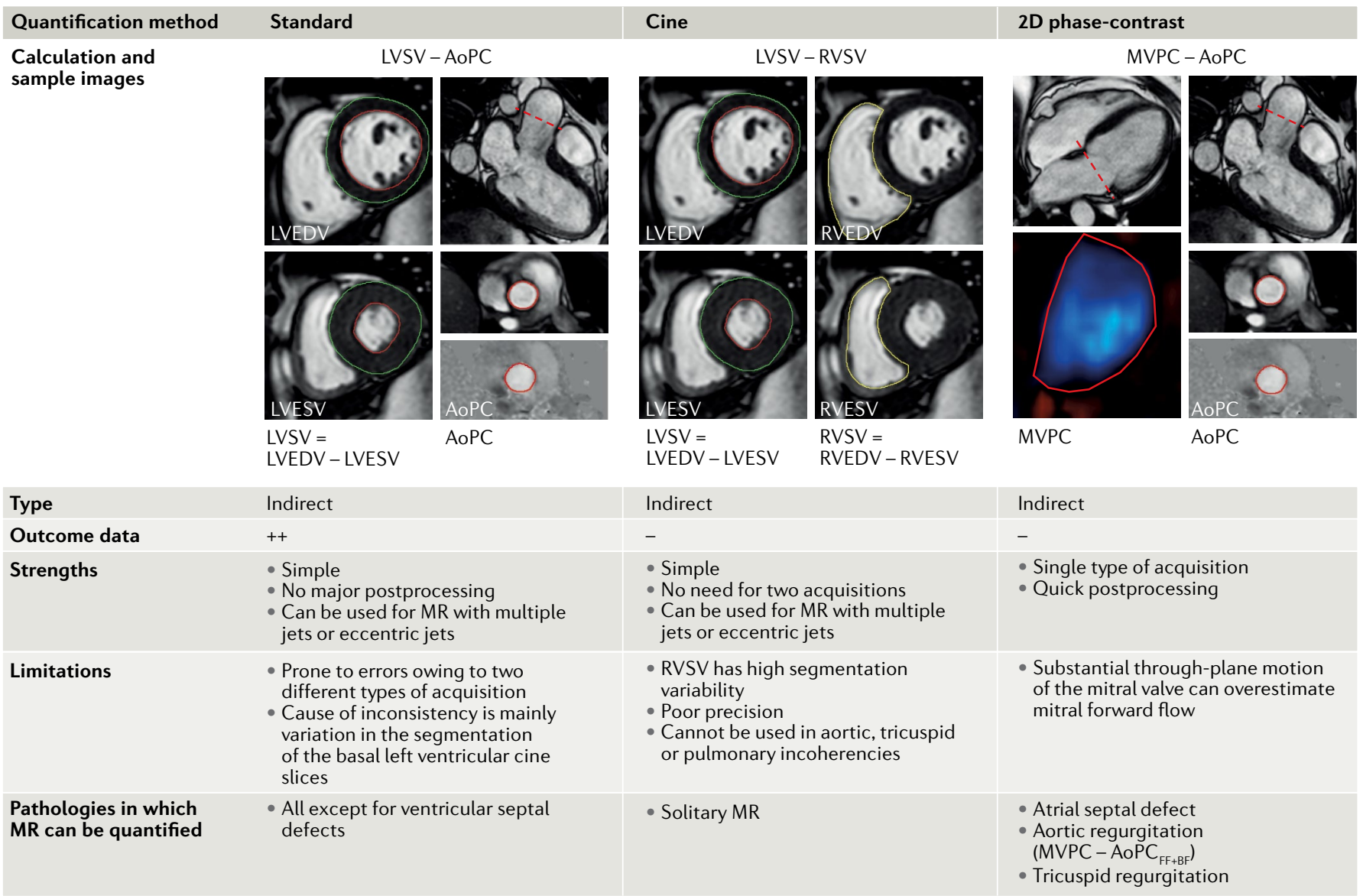

\begin{tabular}{|c|c|c|}
\hline Quantification method & 4D flow & Alternative to standard \\
\hline \multirow[t]{3}{*}{$\begin{array}{l}\text { Calculation and } \\
\text { sample images }\end{array}$} & Direct MR jet & LVSV - PaPC \\
\hline & \multirow{2}{*}{$\begin{array}{l}\begin{array}{l}\text { Retrospective } \\
\text { valve (diastole) } \\
\text { and MR jet tracking } \\
\text { (systole) method }\end{array}\end{array} \rightarrow \begin{array}{l}\text { 3D MR jet } \\
\text { streamline } \\
\text { visualization and } \\
\text { quantification }\end{array}$} & LVESV. \\
\hline & & $\begin{array}{l}\text { LVSV }= \\
\text { LVEDV - LVESV }\end{array}$ \\
\hline Type & Direct & Indirect \\
\hline Outcome data & - & - \\
\hline Strengths & $\begin{array}{l}\text { - Direct quantification } \\
\text { - Can be performed in multiple } \\
\text { pathologies involving shunts and } \\
\text { valvular regurgitations }\end{array}$ & $\begin{array}{l}\text { - Similar strengths to those of the } \\
\text { standard method and less susceptible } \\
\text { to errors from turbulent flow }\end{array}$ \\
\hline Limitations & $\begin{array}{l}\text { - Challenging to perform for MR } \\
\text { with multiple jets }\end{array}$ & $\begin{array}{l}\text { - Similar limitations to those of the } \\
\text { standard method and limited to } \\
\text { patients with no major intracardiac } \\
\text { shunt }\end{array}$ \\
\hline $\begin{array}{l}\text { Pathologies in which } \\
\text { MR can be quantified }\end{array}$ & - All & $\begin{array}{l}\text { - Restrictive ventricular septal defect } \\
\text { and a unidirectional left-to-right } \\
\text { shunt }\end{array}$ \\
\hline
\end{tabular}

Fig. 5 | Main methods of MR quantification by cardiovascular magnetic resonance imaging. Prognostic and diagnostic outcome data are most available for the standard method of quantifying mitral regurgitation (MR) volume $\left(M_{\text {vol }}\right)$, which is left ventricular stroke volume (LVSV) minus aortic phase-contrast forward volume (AoPC). Other methods have particular advantages or disadvantages. In routine clinical practice, crosschecking between methods is recommended. $\mathrm{FF}+\mathrm{BF}$, forward flow plus backward flow; LVEDV, left ventricular end-diastolic volume; LVESV, left ventricular end-systolic volume; MVPC, mitral valve phase-contrast stroke volume; PaPC, pulmonary artery phase-contrast stroke volume; RVEDV, right ventricular end-diastolic volume; RVESV, right ventricular end-systolic volume; RVSV, right ventricular stroke volume. 
with multiple valve lesions or shunt flow as a result of ventricular septal defects.

The third method is, in theory, valid for patients with multiple valve lesions or shunt flow but, in practice, this method often has substantial errors. 2D phase-contrast CMR requires static imaging planes that cannot adapt to through-plane valve motion or the changing location of the mitral valve and the changing direction of inflow or regurgitant jets ${ }^{36,37}$. This method is also susceptible to measuring entrained blood already in the left atrium as part of the regurgitant jet if the imaging slice is too far from the orifice or the region of interest is too large. Furthermore, this method requires two acquisitions, which can be subject to variability.
The 4D-flow CMR, retrospective valve-tracking method (FIG. 6) produces a direct quantification of MR by quantifying flow directly at the valve and is valid in the presence of multiple valve lesions or shunt flow ${ }^{38}$. This approach overcomes the limitations of the third method described above, but acquisition times and postprocessing can be challenging ${ }^{27}$. In retrospective valve tracking, a dynamic reformatted $2 \mathrm{D}$ phase-contrast plane is reconstructed by tracking the mitral annulus over the whole cardiac cycle ${ }^{28,32,38}$. MR jets are quantified by defining a systolic reformatted plane perpendicular to the single jet or individually for multiple jets. Alternatively, if the MR jet is too complex, a reconstructed aortic plane using the retrospective valve-tracking method can be used to
(1) Load 4D flow data and relevant cine
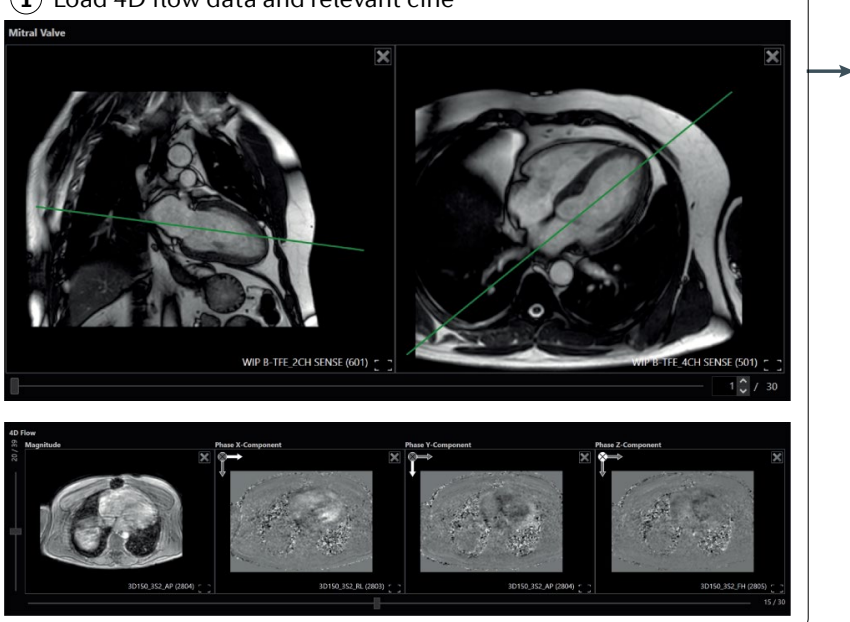

(2) Manual or automatic aliasing correction and offset correction using static tissue

\section{(3) Spatial misalignment correction}

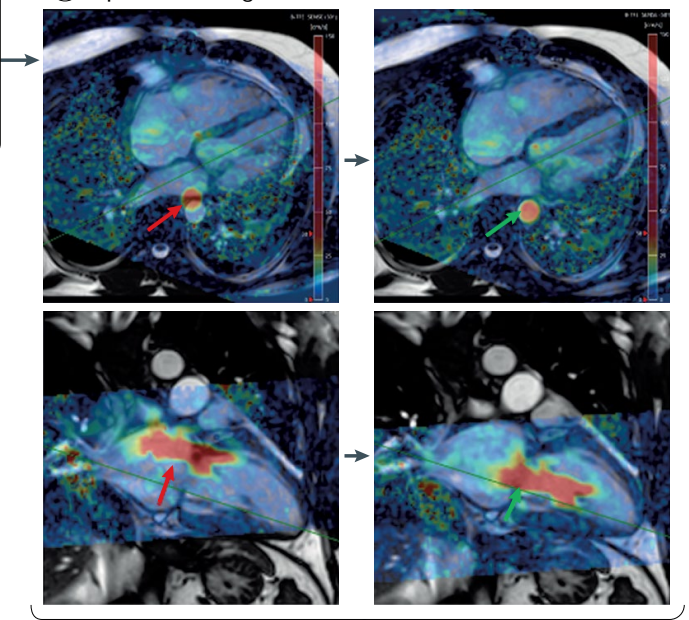

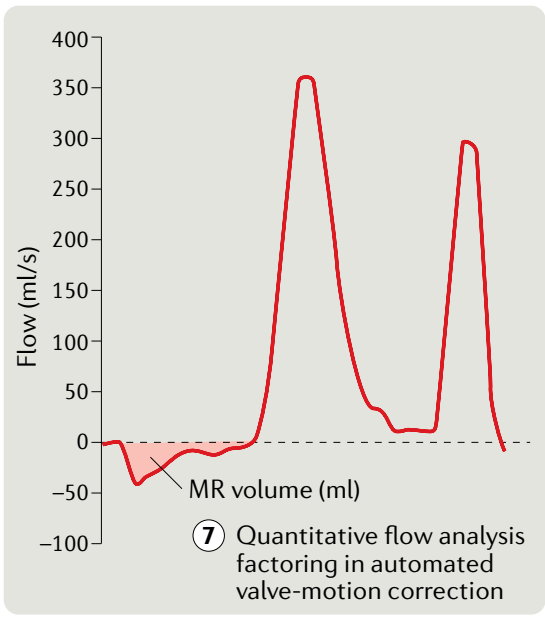

(5) Backward flow segmentation

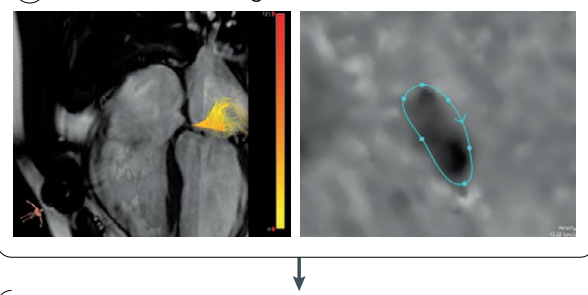

(6) Forward flow segmentation

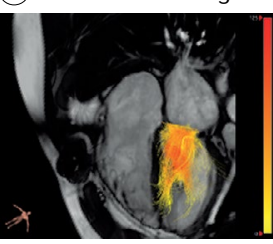

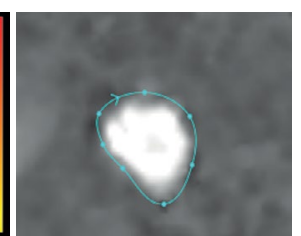

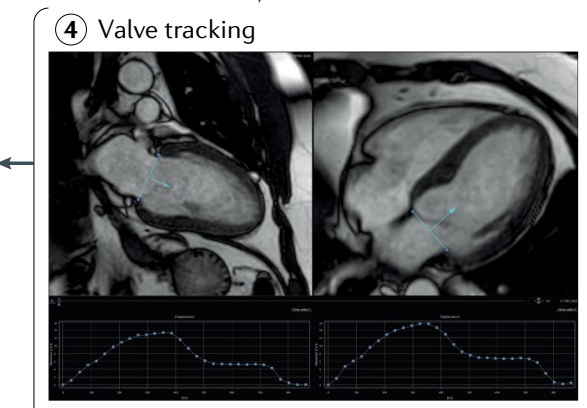

Fig. 6 | 4D-flow cardiovascular magnetic resonance imaging analysis. Step 1: load the two-chamber and four-chamber cardiovascular magnetic resonance imaging cines (upper panel) and 4D phase-contrast flow and 3D phase-contrast data (lower panel). Step 2: depending on the software tool, perform manual or automated aliasing and velocity offset corrections with the use of the static background tissue. Step 3: an attempt to check for spatial misalignment between the cines and the 4D-flow data should be made because the presence of any major misalignment between them will introduce errors in the quantification of valvular flow. The red arrows indicate the spatial misalignment of both the descending aortic flow (in systole) and the mitral inflow (in diastole). The green arrows indicate how this misalignment has been corrected. Step 4: on both the two-chamber and the four-chamber cines, place two landmark points to mark the valve plane.
This marking should be done for the complete cardiac cycle. Software solutions (for example, CAAS software, PIE Medical Imaging, Netherlands) can now automatically track the valve over the complete cardiac cycle. After ensuring the valve is properly tracked, generate a phase-contrast, valvular reformatted plane. Steps 5-6: on the valvular reformatted plane, segment the mitral regurgitant backward flow during left ventricular systole and the forward flow during diastole. The regurgitant plane should be perpendicular to the regurgitant jet. If multiple jets exist, one should make an attempt to evaluate each of them to quantify the total mitral regurgitation (MR). Step 7: quantify valvular forward and backward flow after accounting for the through-plane motion of the valve plane. Similar steps can be used to quantify aortic, tricuspid and pulmonary valvular flows with the use of the 4D-flow data set. 


\section{CONSENSUS STATEMENT}

\section{Box 2 |CMR report for MR assessment}

Include information on the indication for cardiovascular magnetic resonance imaging (CMR)

CMR protocol used for mitral regurgitation (MR) assessment:

- Noncontrast MR assessment

- Contrast MR assessment

- Heart rate and blood pressure

Standard CMR report, including details on:

- Left ventricular and right ventricular regional and global function

- Presence of scarring or infarction on late gadolinium enhancement imaging, with description of myocardial viability and left atrial (LA) and right atrial size

- Any other pathology identified should be described

Mitral valve qualitative assessment

- A detailed description of mitral valve characteristics, including:

- Leaflets: thickened (base, mid or tips), calcification, restricted motion, tethered, bowing, prolapse or flail

- Annulus: annular calcification, fibrosis on late gadolinium enhancement imaging, mitral annular disjunction $>1 \mathrm{~mm}$

- Chordae: thickened, short, restrictive, rupture or tear

- A description of MR jet characteristics:

- Central or eccentric MR jet

- Single or multiple MR jets

- Early, mid, late or pan systolic

MR jet expansion in the left atrium

\section{Quantitative analysis of complete CMR study}

- Dimensions, mass (corrected for body surface area) and function

- Left ventricle: end-diastolic volume, end-systolic volume, stroke volume, ejection fraction and mass

- Right ventricle: end-diastolic volume, end-systolic volume, stroke volume and ejection fraction

- MR (method used to quantify)

- MR volume (ml)

- Regurgitation fraction (\%)

- LA size

- LA volume (ml)

- LA annulus diameter (cm)

\section{Final report conclusions}

- Morphological diagnosis of the aetiology of MR (primary or secondary) and/or Carpentier's functional class of MR

- Degree of MR

- Degree of LA dilatation

- Left ventricular function and degree of dilatation

- Presence, location and degree of myocardial scar or replacement fibrosis

quantify AoPC. This measurement can then be used to quantify MR volume or fraction using the standard LVSV - AoPC method.

Finally, in routine practice, cross-checking MR quantification between methods is useful to reduce uncertainty. Particularly in isolated MR, LVSV - AoPC can easily be cross-checked with LVSV - RVSV or even LVSV - PaPC.

\section{Volumetric cine analysis}

$L V$ and $R V$ volume assessment. $\mathrm{LV}$ and $\mathrm{RV}$ volume quantification is performed according to standard methods ${ }^{33}$. The use of analysis software that allows demarcation of the base of the ventricles on the long-axis images is recommended. LV end-diastolic volume and LVSV are used for the standard method of MR volume calculation (see below) as well as for determining the degree of LV dilatation in response to MR.

LA assessment. LA volumes should be assessed using the biplane area-length method ${ }^{39-41}$. On the basis of the cine long-axis four-chamber and two-chamber views, the contours of the endocardial borders are delineated at end systole (LA diastole). The LA appendage should be included in the atrial volume, but the pulmonary veins should be excluded. $3 \mathrm{D}$ volume methods on the basis of short-axis stacks can be performed and are more accurate than the 2D biplane method, but both the acquisition and postprocessing are much more time consuming.

\section{CMR reporting of MR assessment}

A CMR report for MR assessment should include the standard reporting details described in the CMR standards (BOX 2). The report should include a detailed description of morphological and/or functional characteristics of the mitral leaflets, annulus and chordae tendineae. In addition, a description of MR jet characteristics (such as central or eccentric; single or multiple; early, mid, late or pan systolic) and expansion in the left atrium should be included. The CMR report should mention the method of MR quantification. If any nonstandard method is chosen, a clear reason why it was adopted should be detailed in the report. In the report conclusion, adding the morphological and functional correlates to the aetiology of the MR, including primary or secondary MR and Carpentier's functional class of $\mathrm{MR}$, is helpful.

\section{Reference values to grade MR}

Owing to the lack of a true gold standard, which method quantifies MR severity with the highest degree of accuracy and reliability is unknown. Nonetheless, prognostic studies that demonstrate the superiority of CMR quantification of MR can guide clinical decision-making. Echocardiographic quantification of MR generally shows a bias towards much higher regurgitant volumes than those measured by CMR, so the thresholds that define severity might need to differ according to the imaging technique used. TABLE 3 details the methods and CMR grading used in the three most relevant publications so far. If other methods are used to quantify $\mathrm{MR}$ volume or regurgitation fraction, such as $4 \mathrm{D}$-flow CMR, similar thresholds could be used. However, future large studies are needed to compare different MR quantification methods directly with outcomes to clarify the applicability of the thresholds for different methods.

\section{CMR in clinical pathways}

In routine clinical pathways, if MR is suspected on the basis of clinical signs and symptoms, TTE assessment of MR can determine its aetiology, assess its severity and measure the haemodynamic consequences on the left ventricle. In patients in whom the degree of MR is uncertain, especially between moderate and severe MR, further tests are considered. TOE has been the secondline imaging test not only for clarification of the aetiology but also for assessing the degree of MR. TOE has 
Table 3 | Recommended grading of MR by CMR assessment

\begin{tabular}{|lllll} 
Type of MR & \multicolumn{3}{l}{ Grading of severity } & \\
\cline { 2 - 5 } & Mild & Moderate & Severe & Very severe \\
\hline Primary & $M_{\mathrm{RF}}<20 \%{ }^{\mathrm{a}}$ & $\mathrm{MR}_{\mathrm{RF}}=20-39 \%^{\mathrm{a}}$ & $\mathrm{MR}_{\mathrm{RF}} 40-50 \% ;$ \\
$\mathrm{MR}_{\mathrm{vol}}>55-60 \mathrm{ml}$ & $\mathrm{MR}_{\mathrm{RF}}>50 \%$ \\
\hline Secondary & $\mathrm{MR}_{\mathrm{vol}}<30 \mathrm{ml}$ & $\mathrm{MR}_{\mathrm{vol}}=30-60 \mathrm{ml}$ & $\mathrm{MR}_{\mathrm{vol}} \geq 60 \mathrm{ml}$ & - \\
\hline
\end{tabular}

From REFS ${ }^{12,13,19}$. CMR, cardiovascular magnetic resonance imaging; $M R$, mitral regurgitation; $\mathrm{MR}_{\mathrm{RF}}$, mitral regurgitation fraction; $\mathrm{MR}_{\mathrm{vol}}$, mitral regurgitation volume. ${ }^{\mathrm{N}}$ Not study-based; mainly expert opinion. higher spatial and temporal resolution than either TTE or CMR. However, TOE remains a semi-noninvasive test and relies on successful oesophageal intubation. Although TOE is widely available and is less expensive than CMR, risks are associated with a TOE examination $^{42}$. Overall, with the advent of CMR methods for MR assessment, the real value of TOE will be in providing high-resolution $2 \mathrm{D}$ and $3 \mathrm{D}$ dynamic imaging of the mitral apparatus, mitral valve and scallops to inform and plan surgical intervention, in guiding mitral valve percutaneous interventional procedures and, finally, for intraoperative checks before and after mitral valve intervention. Importantly, MR volume quantified by $3 \mathrm{D}$ TOE has a high level of agreement with that obtained by standard CMR methods ${ }^{43}$.

In patients in whom further assessment of MR severity is needed, we recommend CMR as a second-line noninvasive test. CMR is also recommended in asymptomatic patients with severe MR for further clarification of LV and LA volumes. If a clinical decision is made to 'wait and watch', the focused CMR study proposed in this Consensus Statement can be used to perform longitudinal volumetric and flow assessment to investigate any progression of the MR and associated volume overload on the left ventricle. CMR is not currently recommended for further investigation of vegetation in patients with suspected infective endocarditis-associated MR. This lack of recommendation is mainly because the current spatial resolution of CMR is not high enough, and CMR generates averaged time-resolved images over several cardiac cycles rather than live images. During multidisciplinary meetings involving imaging cardiologist-radiologists, nonimaging cardiologists and surgeons, we recommend actively discussing all imaging options, including
CMR-derived MR metrics, to encourage wider familiarity with CMR methods. In summary, TTE, TOE and CMR are likely to provide complementary information to guide treatment and surveillance in patients with MR.

\section{Future directions}

The evidence that CMR can be used to quantify MR accurately and to predict outcomes ${ }^{12,13}$ makes CMR quantification of MR an attractive tool for future use in randomized controlled trials. The benefit of mitral valve intervention on the basis of the severity of MR (by any modality) has never been studied in a randomized controlled trial, even though mitral valve repair is considered acceptable for asymptomatic patients with a repairable mitral valve according to both European and US guidelines ${ }^{3,5}$.

Future clinical studies are also needed to address whether theoretically more accurate methods of directly quantifying MR, such as retrospective valve tracking using $4 \mathrm{D}$-flow CMR data, are superior to the currently established methods. A need also exists for widespread adoption of robust background flow offset correction methods to provide MR practitioners with confidence in flow quantification. The inaccuracy of quantification in some patients and on some systems is a major barrier to the use of CMR both in MR and in valve disease more generally. Good CMR thresholds for defining the severity of MR are also required, ideally on the basis of outcome data. Existing quantitative thresholds borrowed from echocardiographic data are unsuitable, with wide variation between echocardiography and $\mathrm{CMR}^{12,13}$.

\section{Conclusions}

The assessment of MR by CMR has great utility. CMR is a robust clinical imaging test for the comprehensive assessment of mitral valve morphology and the quantification of MR, with high levels of accuracy. Evidence suggests that CMR can be used to guide and inform clinical outcomes and prognosis in patients with MR. Emerging methods, including 4D-flow CMR, show great promise to improve the precision and accuracy of MR quantification. However, further studies to investigate the clinical benefit of 4D-flow CMR are warranted.

Published online 9 December 2019
1. lung, B. et al. A prospective survey of patients with valvular heart disease in Europe: the Euro Heart Survey on Valvular Heart Disease. Eur. Heart J. 24, 1231-1243 (2003)

2. Nkomo, V. T. et al. Burden of valvular heart diseases: a population-based study. Lancet 368, 1005-1011 (2006).

3. Baumgartner, H. et al. 2017 ESC/EACTS guidelines for the management of valvular heart disease. Eur. Heart $J$. 38, 2739-2791 (2017)

4. Uretsky, S., Argulian, E., Narula, J. \& Wolff, S. D. Use of cardiac magnetic resonance imaging in assessing mitral regurgitation. J. Am. Coll. Cardiol. 71, 547-563 (2018)

5. Doherty, J. U. et al. ACC/AATS/AHA/ASE/ASNC/HRS/ SCAI/SCCT/SCMR/STS 2017 appropriate use criteria for multimodality imaging in valvular heart disease. J. Nucl. Cardiol. 24, 2043-2063 (2017).

6. Stork, A. et al. Assessment of functional anatomy of the mitral valve in patients with mitral regurgitation with cine magnetic resonance imaging: comparison with transesophageal echocardiography and surgical results. Eur. Radiol. 17, 3189-3198 (2007).
7. Suinesiaputra, A. et al. Quantification of LV function and mass by cardiovascular magnetic resonance: multi-center variability and consensus contours. J. Cardiovasc. Magn. Reson. 17, 63 (2015).

8. Han, Y. et al. Cardiovascular magnetic resonance characterization of mitral valve prolapse. JACC Cardiovasc. Imaging 1, 294-303 (2008).

9. Perazzolo Marra, M. et al. Morphofunctional abnormalities of mitral annulus and arrhythmic mitral valve prolapse. Circ. Cardiovasc. Imaging 9 , e005030 (2016).

10. Sturla, F. et al. Dynamic and quantitative evaluation of degenerative mitral valve disease: a dedicated framework based on cardiac magnetic resonance imaging. J. Thorac. Dis. 9, S225-S238 (2017).

11. Ivanov, A. et al. Importance of papillary muscle infarction detected by cardiac magnetic resonance imaging in predicting cardiovascular events. Int. J. Cardiol. 220, 558-563 (2016)

12. Myerson, S. G. et al. Determination of clinical outcome in mitral regurgitation with cardiovascular magnetic resonance quantification. Circulation 133 2287-2296 (2016).
13. Penicka, M. et al. Prognostic implications of magnetic resonance-derived quantification in asymptomatic patients with organic mitral regurgitation. Circulation 137, 1349-1360 (2018)

14. Van De Heyning, C. M. et al. Late gadolinium enhancement CMR in primary mitral regurgitation. Eur. J. Clin. Invest. 44, 840-847 (2014).

15. Kitkungvan, D. et al. Myocardial fibrosis in patients with primary mitral regurgitation with and without prolapse. J. Am. Coll. Cardiol. 72, 823-834 (2018).

16. Edwards, N. C. et al. Quantification of left ventricular interstitial fibrosis in asymptomatic chronic primary degenerative mitral regurgitation. Circ. Cardiovasc Imaging 7, 946-953 (2014).

17. Bharati, S. et al. The conduction system in mitral valve prolapse syndrome with sudden death. Am. Heart J. 101, 667-670 (1981).

18. Dejgaard, L. A. et al. The mitral annulus disjunction arrhythmic syndrome. J. Am. Coll. Cardiol. 72, 1600-1609 (2018).

19. Uretsky, S. et al. Discordance between echocardiography and MRI in the assessment of 
mitral regurgitation severity. J. Am. Coll. Cardiol. 65, 1078-1088 (2015)

20. Flynn, M. et al. Regional wall motion abnormalities and scarring in severe functional ischemic mitral regurgitation: a pilot cardiovascular magnetic resonance imaging study. J. Thorac. Cardiovasc. Surg. 137, 1063-1070.e2 (2009)

21. Kwon, D. H. et al. Predictors and prognostic impact of progressive ischemic mitral regurgitation in patients with advanced ischemic cardiomyopathy: a multimodality study. Circ. Cardiovasc. Imaging 9, e004577 (2016).

22. Kramer, C. M., Barkhausen, J., Flamm, S. D., Kim, R. J. $\&$ Nagel, E. Standardized cardiovascular magnetic resonance (CMR) protocols 2013 update. J. Cardiovasc. Magn. Reson. 15, 91 (2013).

23. Chan, K. M. J. et al. Towards comprehensive assessment of mitral regurgitation using cardiovascular magnetic resonance. J. Cardiovasc Magn. Reson. 10, 61 (2008)

24. Kilner, P. J., Gatehouse, P. D. \& Firmin, D. N. Flow measurement by magnetic resonance: a unique asset worth optimising. J. Cardiovasc. Magn. Reson. 9 723-728 (2007)

25. Gatehouse, P. D. et al. Flow measurement by cardiovascular magnetic resonance: a multi-centre multi-vendor study of background phase offset errors that can compromise the accuracy of derived regurgitant or shunt flow measurements. J. Cardiovasc. Magn. Reson. 12, 5 (2010)

26. Lankhaar, J.-W. et al. Correction of phase offset errors in main pulmonary artery flow quantification. J. Magn. Reson. Imaging 22, 73-79 (2005).

27. Dyverfeldt, P. et al. 4D flow cardiovascular magnetic resonance consensus statement. J. Cardiovasc. Magn. Reson. 17, 72 (2015).

28. Roes, S. D. et al. Flow assessment through four heart valves simultaneously using 3-dimensional 3-directional velocity-encoded magnetic resonance imaging with retrospective valve tracking in healthy volunteers and patients with valvular regurgitation. Invest. Radiol. 44, 669-675 (2009).

29. Fidock, B. et al. A systematic review of 4D-flow MRI derived mitral regurgitation quantification methods. Front. Cardiovasc. Med. 6, 103 (2019).

30. Feneis, J. F. et al. 4D flow MRI quantification of mitral and tricuspid regurgitation: reproducibility and consistency relative to conventional MRI. J. Magn. Reson. Imaging 48, 1147-1158 (2018).

31. Sukpraphrute, B., Chirakarnjanakorn, S. Karaketklang, K., Yindeengam, A. \& Krittayaphong, R. Quantitative measurement of mitral regurgitation: comparison between echocardiography and cardiac magnetic resonance imaging. J. Med. Assoc. Thai. 95, S133-S138 (2012)

32. Garg, P. et al. Comparison of fast acquisition strategies in whole-heart four-dimensional flow cardiac MR: two-center, 1.5 Tesla, phantom and in vivo validation study. J. Magn. Reson. Imaging 47 272-281 (2017)

33. Schulz-Menger, J. et al. Standardized image interpretation and post processing in cardiovascular magnetic resonance: Society for Cardiovascular Magnetic Resonance (SCMR) Board of Trustees Task Force on standardized post processing. J. Cardiovasc. Magn. Reson. 15, 35 (2013).

34. Buchner, S. et al. Cardiovascular magnetic resonance for direct assessment of anatomic regurgitant orifice in mitral regurgitation. Circ. Cardiovasc. Imaging $\mathbf{1}$, 148-155 (2008)

35. Kon, M. W. S., Myerson, S. G., Moat, N. E. \& Pennell, D. J. Quantification of regurgitant fraction in mitral regurgitation by cardiovascular magnetic resonance: comparison of techniques. J. Heart Valve Dis. 13, 600-607 (2004).

36. Calkoen, E. E. et al. Characterization and improved quantification of left ventricular inflow using streamline visualization with 4DFlow MRI in healthy controls and patients after atrioventricular septal defect correction. J. Magn. Reson. Imaging 41, 1512-1520 (2015)

37. Calkoen, E. E. et al. Characterization and quantification of dynamic eccentric regurgitation of the left atrioventricular valve after atrioventricular septal defect correction with 4D Flow cardiovascular magnetic resonance and retrospective valve tracking. J. Cardiovasc. Magn. Reson. 17, 18 (2015).

38. Westenberg, J. J. M. et al. Mitral valve and tricuspid valve blood flow: accurate quantification with 3D velocity-encoded MR imaging with retrospective valve tracking. Radiology $\mathbf{2 4 9}$ 792-800 (2008)
39. Hudsmith, L. E., Petersen, S. E., Francis, J. M., Robson, M. D. \& Neubauer, S. Normal human left and right ventricular and left atrial dimensions using steady state free precession magnetic resonance imaging. J. Cardiovasc. Magn. Reson. 7, 775-782 (2005).

40. Petersen, S. E. et al. Reference ranges for cardiac structure and function using cardiovascular magnetic resonance (CMR) in Caucasians from the UK Biobank population cohort. J. Cardiovasc. Magn. Reson. 19 18 (2017).

41. Peng, J. et al. Normal values of myocardial deformation assessed by cardiovascular magnetic resonance feature tracking in a healthy Chinese population: a multicenter study. Front. Physiol. 9, 1181 (2018).

42. Mathur, S. K. \& Singh, P. Transoesophageal echocardiography related complications. Indian J. Anaesth. 53, 567-574 (2009).

43. Shanks, M. et al. Quantitative assessment of mitral regurgitation: comparison between three-dimensional transesophageal echocardiography and magnetic resonance imaging. Circ. Cardiovasc. Imaging 3 . 694-700 (2010)

44. Heo, R. et al. Clinical implications of threedimensional real-time color Doppler transthoracic echocardiography in quantifying mitral regurgitation: a comparison with conventional two-dimensional methods. J. Am. Soc. Echocardiogr. 30, 393-403.e7 (2017).

45. Harris, A. W. et al. Cardiac magnetic resonance imaging versus transthoracic echocardiography for prediction of outcomes in chronic aortic or mitral regurgitation. Am. J. Cardiol. 119, 1074-1081 (2017).

46. Sachdev, V. et al. Are echocardiography and CMR really discordant in mitral regurgitation? JACC Cardiovasc. Imaging 10, 823-824 (2017).

47. Aplin, M. et al. Cardiac remodelling and function with primary mitral valve insufficiency studied by magnetic resonance imaging. Eur. Heart J. Cardiovasc. Imaging $17,863-870$ (2016)

48. Lopez-Mattei, J. C. et al. Comparative assessment of mitral regurgitation severity by transthoracic echocardiography and cardiac magnetic resonance using an integrative and quantitative approach Am. J. Cardiol. 117, 264-270 (2016).

49. Brugger, $N$. et al. Comparison of three-dimensional proximal isovelocity surface area to cardiac magnetic resonance imaging for quantifying mitral regurgitation. Am. J. Cardiol. 115, 1130-1136 (2015).

50. Choi, J. et al. Differential effect of 3-dimensional color Doppler echocardiography for the quantification of mitral regurgitation according to the severity and characteristics. Circ. Cardiovasc. Imaging 7, 535-544 (2014).

51. Van De Heyning, C. M. et al. Assessment of left ventricular volumes and primary mitral regurgitation severity by $2 \mathrm{D}$ echocardiography and cardiovascular magnetic resonance. Cardiovasc. Ultrasound 11, 46 (2013).

52. Thavendiranathan, P. et al. Quantification of chronic functional mitral regurgitation by automated 3-dimensional peak and integrated proximal isovelocity surface area and stroke volume techniques using real-time 3-dimensional volume color Doppler echocardiography: in vitro and clinical validation. Circ. Cardiovasc. Imaging 6, 125-133 (2013).

53. Son, J.-W. et al. Automated quantification of mitral regurgitation by three dimensional real time full volume color Doppler transthoracic echocardiography: a validation with cardiac magnetic resonance imaging and comparison with two dimensional quantitative methods. J. Cardiovasc. Ultrasound 21 81 (2013)

54. Reddy, S. T. et al. Evaluation of cardiac valvular regurgitant lesions by cardiac MRI sequences: comparison of a four-valve semi-quantitative versus quantitative approach. J. Heart Valve Dis. 22, 491-499 (2013)

55. Cawley, P. J. et al. Prospective comparison of valve regurgitation quantitation by cardiac magnetic resonance imaging and transthoracic echocardiography. Circ. Cardiovasc. Imaging 6, 48-57 (2013).

56. Hamada, S. et al. Comparison of accuracy of mitral valve regurgitation volume determined by threedimensional transesophageal echocardiography versus cardiac magnetic resonance imaging. Am. J. Cardiol. 110, 1015-1020 (2012)

57. Skaug, T. R. et al. Quantification of mitral regurgitation using high pulse repetition frequency three- dimensional color Doppler. J. Am. Soc. Echocardiogr. 23, 1-8 (2010)

58. Myerson, S. G., Francis, J. M. \& Neubauer, S Direct and indirect quantification of mitral regurgitation with cardiovascular magnetic resonance, and the effect of heart rate variability. MAGMA 23, 243-249 (2010).

59. Hellgren, L. et al. Severe mitral regurgitation relations between magnetic resonance imaging, echocardiography and natriuretic peptides. Scand. Cardiovasc. J. 42, 48-55 (2008).

60. Gabriel, R. S. et al. Mapping of mitral regurgitant defects by cardiovascular magnetic resonance in moderate or severe mitral regurgitation secondary to mitral valve prolapse. J. Cardiovasc. Magn. Reson. 10, 16 (2008)

61. Gelfand, E. V. et al. Severity of mitral and aortic regurgitation as assessed by cardiovascular magnetic resonance: optimizing correlation with Doppler echocardiography. J. Cardiovasc. Magn. Reson. 8, 503-507 (2006).

62. Kizilbash, A. M. et al. Comparison of quantitative Doppler with magnetic resonance imaging for assessment of the severity of mitral regurgitation. Am. J. Cardiol. 81, 792-795 (1998).

63. Hundley, W. G. et al. Magnetic resonance imaging assessment of the severity of mitral regurgitation. Comparison with invasive techniques. Circulation 92, 1151-1158 (1995).

64. Polte, C. L., Gao, S. A., Johnsson, Å. A., Lagerstrand, K. M. \& Bech-Hanssen, O. Characterization of chronic aortic and mitral regurgitation undergoing valve surgery using cardiovascular magnetic resonance. Am. J. Cardiol. 119, 2061-2068 (2017).

65. Buck, T. et al. Effect of dynamic flow rate and orifice area on mitral regurgitant stroke volume quantification using the proximal isovelocity surface area method. J. Am. Coll. Cardiol. 52, 767-778 (2008).

66. Fujita, N. et al. Quantification of mitral regurgitation by velocity-encoded cine nuclear magnetic resonance imaging. J. Am. Coll. Cardiol. 23 951-958 (1994).

67. Kamphuis, V. P. et al. Automated cardiac valve tracking for flow quantification with four-dimensional flow MRI. Radiology 290, 70-78 (2019).

68. Marsan, N. A. et al. Quantification of functional mitral regurgitation by real-time 3D echocardiography: comparison with 3D velocity-encoded cardiac magnetic resonance. JACC Cardiovasc. Imaging 2 , 1245-1252 (2009).

69. Gorodisky, L., Agmon, Y., Porat, M., Abadi, S \& Lessick, J. Assessment of mitral regurgitation by 3-dimensional proximal flow convergence using magnetic resonance imaging: comparison with echoDoppler. Int. J. Cardiovasc. Imaging 34, 793-802 (2018).

70. Uretsky, S. et al. Quantification of left ventricular remodeling in response to isolated aortic or mitral regurgitation. J. Cardiovasc. Magn. Reson. 12, 32 (2010).

71. Heitner, J. et al. Clinical application of cine-MRI in the visual assessment of mitral regurgitation compared to echocardiography and cardiac catheterization. PLOS ONE 7, e40491 (2012).

72. Ozdogan, O. et al. Evaluation of the severity of mitral regurgitation by the use of signal void in magnetic resonance imaging. Echocardiography 26 1127-1135 (2009).

73. Aurigemma, G., Reichek, N., Schiebler, M. \& Axel, L. Evaluation of mitral regurgitation by cine magnetic resonance imaging. Am. J. Cardiol. 66, 621-625 (1990).

74. Pflugfelder, P. W. et al. Noninvasive evaluation of mitral regurgitation by analysis of left atrial signal loss in cine magnetic resonance. Am. Heart J. 117 1113-1119 (1989).

\section{Acknowledgements}

C.B.-D. is partly supported by the National Institute for Health Research (NIHR) Biomedical Research Centre at University Hospitals Bristol National Health Service (NHS) Foundation Trust and the University of Bristol. The views expressed in this publication are those of the authors and not necessarily those of the NHS, the NIHR or the Department of Health and Social Care.

\section{Author contributions}

P.G. and S.G.M. conceived the need for the article. P.G., A.J.S., L.Z., C.-J.C., T.E. and J.W. drafted the initial version. M.D.H., 


\section{CONSENSUS STATEMENT}

C.B.-D., J.J.B. and S.G.M. provided expert critical input to the content. All the authors read and approved the manuscript.

Competing interests

The authors declare no competing interests.

\section{Peer review information}

Nature Reviews Cardiology thanks F. Maisano, M. Motwan and the other, anonymous, reviewer(s) for their contribution to the peer review of this work.
Publisher's note

Springer Nature remains neutral with regard to jurisdictional claims in published maps and institutional affiliations.

(c) (i)

Open Access This article is licensed under Creative Commons Attribution 4.0 International License, which permits use sharing, adaptation, distribution and reproduction in any medium or format, as long as you give appropriate credit to the original author(s) and the source, provide a link to the
Creative Commons licence, and indicate if changes were made. The images or other third party material in this article are included in the article's Creative Commons licence, unless indicated otherwise in a credit line to the material. If material is not included in the article's Creative Commons licence and your intended use is not permitted by statutory regulation or exceeds the permitted use, you will need to obtain permission directly from the copyright holder. To view a copy of this licence, visit http://creativecommons.org/licenses/by/4.0/.

(c) The Author(s) 2019 